\title{
A randomized trial of online single-session interventions for adolescent depression during COVID-19
}

\author{
Jessica L. Schleider $\oplus^{\circledR}$, Michael C. Mullarkey $\oplus^{1}$, Kathryn R. Fox $\oplus^{2}{ }^{2}$, Mallory L. Dobias $\oplus^{1}$, \\ Akash Shroff ${ }^{1}{ }^{1}$, Erica A. Hart ${ }^{2}$ and Chantelle A. Roulston ${ }^{1}$
}

The COVID-19 pandemic has potentially increased the risk for adolescent depression. Even pre-pandemic, $<\mathbf{5 0} \%$ of youth with depression accessed care, highlighting needs for accessible interventions. Accordingly, this randomized controlled trial (ClinicalTrials.gov: NCT04634903) tested online single-session interventions (SSIs) during COVID-19 in adolescents with elevated depression symptoms $(N=2,452$, ages 13-16). Adolescents from all 50 US states, recruited via social media, were randomized to one of three SSIs: a behavioural activation SSI, an SSI teaching that traits are malleable and a supportive control. We tested each SSI's effects on post-intervention outcomes (hopelessness and agency) and three-month outcomes (depression, hopelessness, agency, generalized anxiety, COVID-19-related trauma and restrictive eating). Compared with the control, both active SSIs reduced three-month depressive symptoms (Cohen's $\boldsymbol{d}=\mathbf{0 . 1 8}$ ), decreased post-intervention and three-month hopelessness $(d=0.16-0.28)$, increased post-intervention agency $(d=0.15-0.31)$ and reduced three-month restrictive eating $(d=0.12-17)$. Several differences between active SSIs emerged. These results confirm the utility of free-of-charge, online SSIs for high-symptom adolescents, even in the high-stress COVID-19 context.

\begin{abstract}
n the early months of 2020, the COVID-19 pandemic swiftly and profoundly transformed the lives of youths nationwide. School closures affecting $>50$ million students led to isolation and the disruption of educational and social-emotional supports; simultaneously, families grappled with collective trauma and economic recession ${ }^{1}$. Together, these stressors might increase risk for adolescent depression-already the world's leading cause of disability in young people $\mathrm{e}^{2-4}$. Even before the pandemic, fewer than $50 \%$ of adolescents with depression accessed services ${ }^{5,6}$; among those who did, $40-65 \%$ have failed to respond ${ }^{7,8}$. Newfound financial strain may further preclude families' capacity to afford treatment for their children. A generation of youth exposed to unprecedented psychosocial adversity is thus poised to fall through the cracks of the mental health-care system. It is critical to identify effective, scalable strategies to reduce adolescent depression, both during and beyond COVID-19. Accordingly, in a nationwide randomized controlled trial, we tested whether two self-guided, online, single-session interventions (SSIs) for adolescent depression-one teaching a growth mindset of personality (the belief that personal traits and symptoms can change) and another teaching behavioural activation (the practice of managing one's mood via engagement in valued, enjoyable activities)—could reduce hopelessness, strengthen perceived agency and mitigate symptoms of depression, anxiety and COVID-19-related trauma in high-symptom youth, versus a supportive control.

Well-powered trials of brief, focused and rapidly scalable interventions may overcome long-standing challenges to reducing adolescent depression-namely, the challenges of limited potency of existing treatments and of low accessibility in predominant modes of care. The difficulties underlying limited treatment potency are thought to reflect depression's heterogeneity ${ }^{9,10}$. Diagnostic criteria for depression place youths with five of nine diverse symptoms
\end{abstract}

(such as activity withdrawal, fatigue and hopelessness) into a single category including $>1,400$ possible symptom combinations ${ }^{9}$. This heterogeneity has spurred the creation of interventions that target wide-ranging difficulties, some of which may be unrelated to an individual's needs-suggesting the utility of highly focused, targeted interventions rather than those characterized by extreme comprehensiveness (for example, cognitive behavioural therapy) ${ }^{11,12}$. Large-scale trials can rigorously and definitively gauge the promise of treatments that are designed for brevity, containing just one or two treatment elements rather than ten or more separate modules.

Large trials of brief interventions may also reveal solutions to the low accessibility of many depression interventions. This low accessibility stems from the formats of predominant treatments, which span many weeks and are intended for delivery in brick-and-mortar clinics by highly trained clinicians, creating major dissemination barriers ${ }^{13}$. Furthermore, up to $59 \%$ of youths who do access mental health treatment drop out prematurely, compounding the challenges posed by provider scarcity ${ }^{14,15}$. Testing brief treatments deliverable by flexible means is a key component of solving this access-to-care crisis.

The need for accessible, brief and targeted adolescent depression interventions has grown during the pandemic. Before the pandemic, research demonstrated that environmental stress, family instability and social isolation can compound risk for youth depression-and each of these stressors has intensified since the pandemic's emergence ${ }^{2-4,7}$. It is thus possible that risk for youth depression may increase in the upcoming years, yet access to effective services remains scarce. It is as critical as ever to rigorously evaluate rapidly scalable, low-cost strategies to reduce youth depression.

SSIs for youth depression may offer a sustainable path towards this goal. SSIs include core elements of evidence-based treatments, but their brevity makes them easier to disseminate to diverse settings ${ }^{16}$.

'Department of Psychology, Stony Brook University, Stony Brook, NY, USA. ²Department of Psychology, University of Denver, Denver, CO, USA.

凶e-mail: jessica.schleider@stonybrook.edu 
In a meta-analysis of 50 randomized trials, SSIs reduced diverse youth mental health difficulties (mean effect size Hedges $g=0.32$ ), including self-administered SSIs (for example, web-based SSIs; mean effect size Hedges $g=0.32)^{17}$. To date, systematic and narrative reviews have identified two types of SSI that have reduced adolescent depression symptoms ${ }^{16,17}$. The first is a growth mindset (GM) SSI, an online programme teaching that personal traits are malleable, which has prevented and reduced adolescent depression in multiple trials ${ }^{18-22}$. As examples, a GM-SSI led to nine-month depression symptom reductions in high-symptom adolescents, versus a supportive control (Cohen's $d=0.60$ and 0.32 per parent and youth reports $)^{19}$. In two school-based randomized trials, GM-SSIs reduced adolescent depressive symptoms versus active controls from four- to nine-month follow-ups ${ }^{18,21,22}$. The second type, a behavioural activation (BA) SSI, promotes values-based activity engagement to elicit pleasure and accomplishment ${ }^{23-25}$. In randomized trials, therapist-guided BA-SSIs have reduced depressive symptoms in moderately depressed adolescents across two weeks $(d=1.61)$ and one month $(d=0.57)^{23,24}$. Furthermore, in an open trial, high-symptom adolescents reported increases in perceived problem-solving ability and agency after completing an online, self-guided BA-SSI; they also endorsed the BA-SSI's overall acceptability and helpfulness ${ }^{20}$. Notably, the GM-SSI and BA-SSI are the only two fully self-guided, single-session, digital interventions that have shown acceptability and (at minimum) short-term utility among US adolescents with elevated depressive symptoms. However, their relative effectiveness has yet to be rigorously assessed, creating a need to understand their potentially differential promise as widely scaled supports for youth depression and related difficulties.

During the pandemic, large trials of multiple online SSIs can elucidate their relative utility for depression, as well as for other mental health difficulties, such as COVID-19-related trauma or anxiety symptoms. Indeed, proximal targets addressed by both the BA and GM SSIs, such as hope ${ }^{20}$, relate to depression and anxiety alike. Likewise, interventions designed to increase perceived agency ${ }^{22}$ and active problem-solving ${ }^{26}$ have reduced both anxiety and depression in youth. SSIs targeting agency and hope (such as the BA-SSI and GM-SSI) may thus help reduce depression symptom severity, anxiety symptoms and trauma symptoms linked to COVID-19and potentially other forms of psychopathology not directedly targeted by the SSIs (for example, restrictive eating). SSIs circumvent many common treatment access barriers: they require no therapist, are completable from any location and are $<30$ minutes in length, eliminating premature dropout concerns. Moreover, online SSIs hold advantages even over other digital interventions, which tend to require sustained effort and repeated use, leading to low engagement and rapid dropout ${ }^{27}$. Online SSIs thus offer a unique opportunity for rapid, large-scale tests of accessible depression interventions while the pandemic remains underway.

We therefore conducted a nationwide randomized controlled trial comparing the utility of online SSIs for adolescent depression during the COVID-19 pandemic ( $N=2,452$ youth with elevated depressive symptoms, ages 13-16). The trial had three primary aims. Aim 1 was to test whether online, self-guided SSIs improved proximal, clinically relevant targets (for example, hopelessness and perceived agency, which have predicted longer-term SSI responses $^{22}$ ) and three-month depressive symptoms (primary outcome), relative to an active control. Aim 2 was to test whether the GM-SSI or the BA-SSI-currently, the only two self-guided, digital SSIs that have shown high acceptability in youths experiencing depressive symptoms-proved more impactful in this context. Aim 3 was to test whether either SSI improved secondary, clinically relevant outcomes (COVID-19-related trauma, hopelessness, agency and generalized anxiety) versus the control across three months. We also conducted post hoc exploratory analyses to test intervention impacts on difficulties not directly targeted by the SSIs (restrictive eating). Adolescents recruited from across the United States via social media were randomized to one of three self-guided SSIs: a BA-SSI, designed to address activity withdrawal and low agency; a GM-SSI, designed to target hopelessness; and a supportive control. We tested each SSI's relative benefits, versus the control, across three months of the COVID-19 pandemic (November 2020-March 2021). Notably, the trial took place approximately eight months after school closures and social distancing mandates were first imposed in the United States but before the COVID-19 vaccine was publicly available. The trial thus took place at a time when pandemic-related conditions were still evolving and unpredictable in many US regions and when some adolescents might have begun to adjust to lifestyle changes and norms.

\section{Results}

Sample characteristics. Table 1 presents characteristics of the 2,452 adolescents randomized to an SSI, and Fig. 1 (CONSORT diagram) presents participant retention throughout the study. Per a cut-off score of $\geq 10$ for the short-form Children's Depression Inventory 2 $(\mathrm{CDI}-\mathrm{SF})^{28}, 86.17 \%$ of youths reported clinically elevated depressive symptoms at baseline. This sample was thus comparable to those in treatment studies, wherein a majority of youths' symptom severity scores exceed standard cut-offs. No group differences emerged on demographic factors or baseline depressive symptoms, indicating successful randomization. The participants were $88.09 \%$ female (sex assigned at birth) and 3.75\% American Indian, $12.64 \%$ Asian, 10.48\% Black, 1.59\% Native Hawaiian, 19.21\% Hispanic and $66.56 \%$ White (these categories were not mutually exclusive). Approximately $80 \%$ of the adolescents identified with a sexual minority identity, and the participating youths lived in all 50 US states (Supplementary Fig. 2 illustrates the participants' locations throughout the United States).

Intervention acceptability. Adolescents completed the baseline survey in 43.31 minutes (median), including pre-SSI questionnaires, one randomly assigned SSI and post-SSI surveys. Among adolescents randomized to an SSI, 83.76\% completed it in full (range across conditions, $80.32-89.16 \%$ ). Youths found both active SSIs and the placebo control SSI acceptable across all Program Feedback Scale (PFS) items (the item-level means were all above 3.50/5; see Table 2 for the item-level ratings by condition).

Differential SSI completion and follow-up survey completion. Assumptions were met for the interpretation of two-tailed tests regarding differential SSI and follow-up survey completion. Participants in Project ABC dropped out significantly less $\left(\chi^{2}(2)=41.47, P<.001,11.08 \%\right)$ during the intervention than participants in Project Personality $(19.68 \%)$ or the Sharing Feelings placebo $(22.86 \%)$. There were no significant differences in who initiated the three-month follow-up across conditions $\left(\chi^{2}(2)=2.14\right.$; $P=0.34$; Project ABC, 37.39\%; Project Personality, 38.02\%; Sharing Feelings placebo, 40.71\%). Logistic regressions using baseline depression symptom and demographic data were unable to predict whether participants would drop out during the intervention (AUC, 0.51) or at three-month follow-up (AUC, 0.56) better than chance (see the analyses on the Open Science Framework for further details: https://osf.io/8mk6x/).

Depression severity outcomes. Here we describe the pre-registered primary outcome: depression severity (see Table 3 for descriptives for the primary and secondary outcome variables at baseline, post-intervention and follow-up; Fig. 2 for visualization of the results for depression; and Supplementary Table 1 for the full results of the regression analysis, for which all assumptions for interpretation were met). Compared with adolescents randomized to the 
Table 1 | Sample characteristics

\begin{tabular}{|c|c|c|c|}
\hline \multirow[b]{2}{*}{ Demographics } & \multicolumn{3}{|c|}{ Treatment received } \\
\hline & Placebo control, $N=818^{a}$ & Project $A B C, N=821^{a}$ & Project Personality, $N=813^{a}$ \\
\hline \multicolumn{4}{|l|}{ Race/ethnicity } \\
\hline Asian including Asian Desi & $101(12 \%)$ & $109(13 \%)$ & $100(12 \%)$ \\
\hline Hispanic/Latinx & $159(19 \%)$ & $164(20 \%)$ & $148(18 \%)$ \\
\hline Black/African American & $85(10 \%)$ & $84(10 \%)$ & $88(11 \%)$ \\
\hline Other & $19(2.3 \%)$ & $17(2.1 \%)$ & $11(1.4 \%)$ \\
\hline Prefer not to answer & $9(1.1 \%)$ & $5(0.6 \%)$ & $10(1.2 \%)$ \\
\hline \multicolumn{4}{|l|}{ Gender identity } \\
\hline Agender & $19(2.3 \%)$ & $12(1.5 \%)$ & $23(2.8 \%)$ \\
\hline Non-binary & $128(16 \%)$ & $127(15 \%)$ & $129(16 \%)$ \\
\hline Two-spirited & $5(0.6 \%)$ & $5(0.6 \%)$ & $6(0.7 \%)$ \\
\hline Transgender-female to male & $59(7.2 \%)$ & $59(7.2 \%)$ & $62(7.6 \%)$ \\
\hline Trans female/trans-feminine & $7(0.9 \%)$ & $9(1.1 \%)$ & $9(1.1 \%)$ \\
\hline Trans male/trans-masculine & $67(8.2 \%)$ & $65(7.9 \%)$ & $64(7.9 \%)$ \\
\hline Gender expansive & $7(0.9 \%)$ & $11(1.3 \%)$ & $9(1.1 \%)$ \\
\hline Third gender & $2(0.2 \%)$ & $1(0.1 \%)$ & $3(0.4 \%)$ \\
\hline Genderqueer & $46(5.6 \%)$ & $45(5.5 \%)$ & $49(6.0 \%)$ \\
\hline Transgender-male to female & $6(0.7 \%)$ & $3(0.4 \%)$ & $6(0.7 \%)$ \\
\hline Other & $2(0.2 \%)$ & $12(1.5 \%)$ & $7(0.9 \%)$ \\
\hline Prefer not to say & $6(0.7 \%)$ & $5(0.6 \%)$ & $9(1.1 \%)$ \\
\hline \multicolumn{4}{|l|}{ Sexual orientation } \\
\hline Asexual & $39(4.8 \%)$ & $46(5.6 \%)$ & $38(4.7 \%)$ \\
\hline Bisexual & $228(28 \%)$ & $230(28 \%)$ & $220(27 \%)$ \\
\hline Gay/lesbian/homosexual & $86(11 \%)$ & $76(9.3 \%)$ & $91(11 \%)$ \\
\hline Heterosexual/straight & $172(21 \%)$ & $161(20 \%)$ & $165(20 \%)$ \\
\hline Do not use a label & $52(6.4 \%)$ & $41(5.0 \%)$ & $47(5.8 \%)$ \\
\hline Do not want to respond & $3(0.4 \%)$ & $5(0.6 \%)$ & $2(0.2 \%)$ \\
\hline Other/not listed & $32(3.9 \%)$ & $23(2.8 \%)$ & $38(4.7 \%)$ \\
\hline Pansexual & $87(11 \%)$ & $98(12 \%)$ & $79(9.7 \%)$ \\
\hline Queer & $49(6.0 \%)$ & $47(5.7 \%)$ & $51(6.3 \%)$ \\
\hline Unsure/questioning & $70(8.6 \%)$ & $94(11 \%)$ & $82(10 \%)$ \\
\hline Baseline CDI-SF sum score $(0-24)$ & $14.31(4.12)$ & $14.15(4.06)$ & $14.22(4.13)$ \\
\hline
\end{tabular}

The demographic data are presented as $N(\%)$; the baseline scores are presented as mean (s.d.).

control SSI, adolescents randomized to the BA-SSI reported significant decreases in depression symptom severity from pre-intervention to three-month follow-up $\left(t(1,673)=-3.62 ; P_{\text {adj }}<0.001 ; d=0.18\right.$; $95 \%$ confidence interval (CI), 0.08, 0.28). Likewise, adolescents randomized to the GM-SSI reported significant decreases in depression symptom severity from pre-intervention to three-month follow-up, relative to control-group adolescents $(t(1,629)=-3.53$; $\left.P_{\text {adj }}<0.001 ; d=0.18 ; 95 \% \mathrm{CI}, 0.08,0.27\right)$. Adolescents randomly 

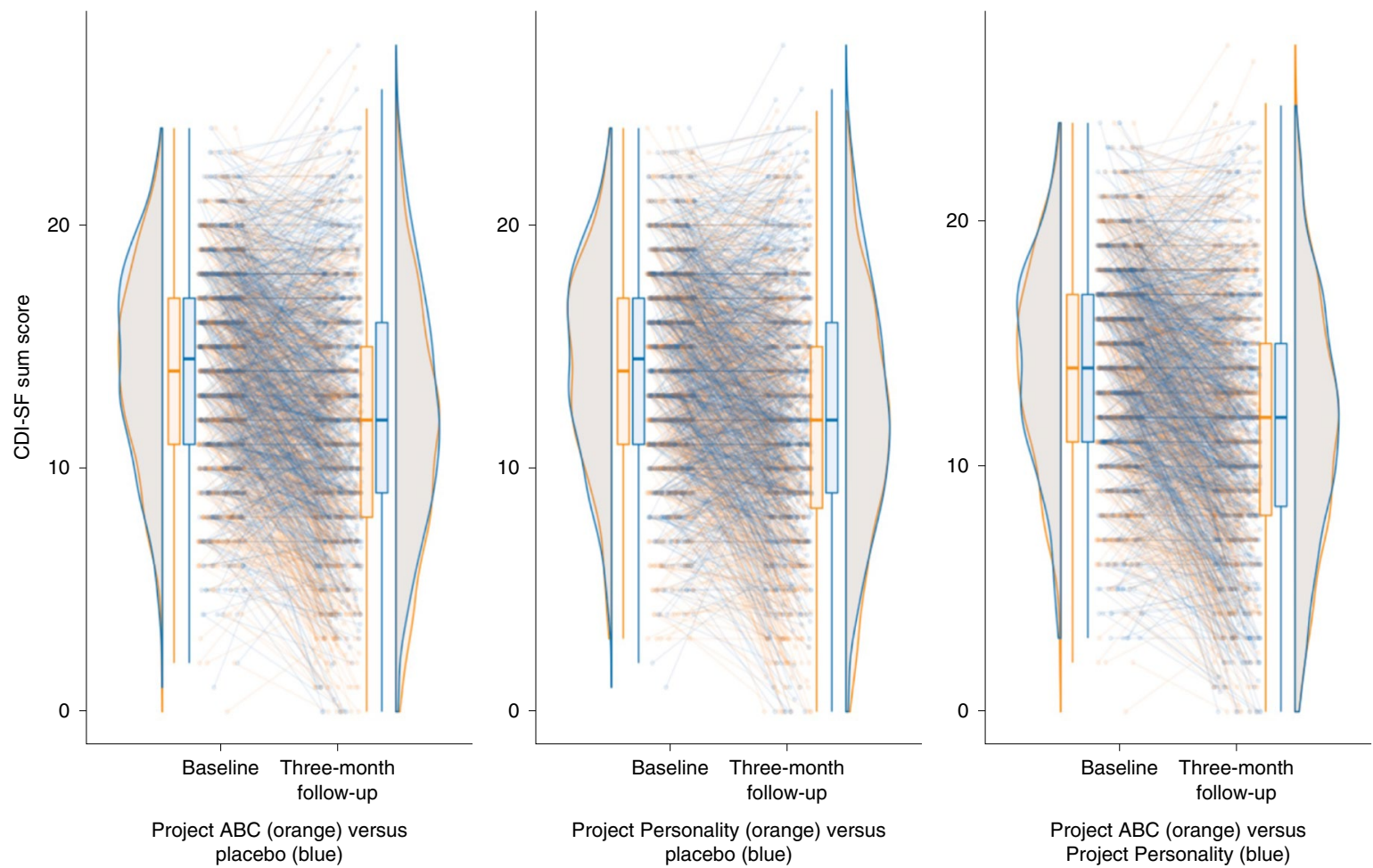

Fig. 1 | Depression symptom severity at baseline and three-month follow-up for adolescents in each intervention condition. The interventions were Project ABC/BA-SSI ( $=818)$, Project Personality/GM-SSI $(N=821)$ and the Sharing Feelings Project/placebo control $(N=818)$. Adolescents randomized to the BA-SSI reported significant decreases in depression symptom severity from pre-intervention to three-month follow-up compared to adolescents in the placebo control $\left(P_{\mathrm{adj}}<0.001\right)$; adolescents randomized to the GM-SSI also reported significant decreases relative to control-group adolescents $\left(P_{\text {adj }}<0.001\right)$. Adolescents assigned to the BA-SSI and GM-SSI did not significantly differ in their depression symptom reductions $\left(P_{\text {adj }}=0.845\right)$.

Table 2 | PFS item-level means and standard deviations by intervention condition, among adolescents who completed each SSI in full

\begin{tabular}{|c|c|c|c|}
\hline \multirow[b]{2}{*}{$\begin{array}{l}\text { PFS items (range } \\
\text { across items, 1-5) }\end{array}$} & \multicolumn{3}{|c|}{ SSI received } \\
\hline & $\begin{array}{l}\text { Placebo } \\
\text { control, } \\
N=630^{\text {a }}\end{array}$ & $\begin{array}{l}\text { Project ABC } \\
\text { (BA-SSI), } \\
N=729^{a}\end{array}$ & $\begin{array}{l}\text { Project } \\
\text { Personality } \\
\text { (GM-SSI) } \\
N=653^{\mathrm{a}}\end{array}$ \\
\hline Enjoyed & $3.80(0.79)$ & $3.93(0.73)$ & $3.83(0.77)$ \\
\hline Understood & $4.54(0.56)$ & $4.46(0.59)$ & $4.48(0.64)$ \\
\hline Easy to Use & $4.46(0.64)$ & $4.42(0.64)$ & $4.39(0.68)$ \\
\hline Tried My Hardest & $4.45(0.65)$ & $4.42(0.69)$ & $4.45(0.67)$ \\
\hline $\begin{array}{l}\text { Helpful to Other } \\
\text { Kids }\end{array}$ & $4.24(0.81)$ & $4.30(0.76)$ & $4.20(0.83)$ \\
\hline $\begin{array}{l}\text { Would } \\
\text { Recommend to a } \\
\text { Friend }\end{array}$ & $3.97(0.95)$ & $4.12(0.89)$ & $3.93(1.00)$ \\
\hline $\begin{array}{l}\text { Agree with } \\
\text { Message }\end{array}$ & $4.50(0.62)$ & $4.54(0.57)$ & $4.47(0.70)$ \\
\hline \multicolumn{4}{|c|}{$\begin{array}{l}\text { aThe results are shown as mean (s.d.). Most item-level acceptability ratings did not differ by } \\
\text { condition. Exceptions were that youths reported (1) enjoying the BA-SSI and placebo more than } \\
\text { the GM-SSI, (2) understanding the placebo more than the BA-SSI and (3) being more likely to } \\
\text { recommend the BA-SSI to a friend, versus both other conditions. }\end{array}$} \\
\hline
\end{tabular}

assigned to the BA-SSI and GM-SSI did not significantly differ in their depression symptom reductions from pre-intervention to three-month follow-up $\left(t(1,632)=-0.20 ; P_{\text {adj }}=0.845 ; d=-0.01\right.$; 95\% CI, $-0.11,0.09$ ).
Regarding within-group effects (presented here to contextualize within-group symptom changes across conditions, not as indicators of efficacy), adolescents randomized to each SSI condition reported significant reductions in depressive symptom severity from baseline to three-month follow-up; within-group reductions were numerically larger for the BA-SSI $(t(820)=-9.62 ; P<0.001$; $d=-0.47 ; 95 \% \mathrm{CI},-0.54,-0.39)$ and the GM-SSI $(t(812)=-12.29$; $P<0.001 ; d=-0.43 ; 95 \% \mathrm{CI},-0.50,-0.36)$ than for the control $(t(817)=-12.29 ; P<0.001 ; d=-0.34 ; 95 \% \mathrm{CI},-0.41,-0.27)$.

Hopelessness and agency outcomes. We investigated hopelessness and agency as pre-registered secondary outcomes at post-intervention and as exploratory secondary outcomes at follow-up (see Supplementary Tables 4-7 for the full results of the regression analyses, for which all assumptions for interpretation were met). Compared with adolescents randomized to the supportive control, adolescents randomized to the BA-SSI reported significant reductions in hopelessness at post-intervention $\left(t(1,637)=-5.22 ; P_{\text {adj }}<0.001 ; d=0.26 ; 95 \%\right.$ CI, $\left.0.16,0.36\right)$ and three-month follow-up $(t(1,637)=-3.49 ; P<0.001 ; d=0.17 ; 95 \%$ $\mathrm{CI}, 0.08,0.27)$. The BA-SSI also led to increases in perceived agency at post-intervention $\left(t(1,637)=6.20 ; P_{\mathrm{adj}}<0.001 ; d=-0.31 ; 95 \% \mathrm{CI}\right.$, $-0.40,-0.21)$ but not follow-up $(t(1,637)=1.57 ; P=0.12 ; d=0.08$; $95 \% \mathrm{CI},-0.02,0.17)$ versus the control. Likewise, adolescents randomized to the GM-SSI reported significant reductions in hopelessness at post-intervention $\left(t(1,629)=-5.80 ; P_{\text {adj }}<0.001 ; d=0.28\right.$; $95 \% \mathrm{CI}, 0.18,0.38)$ and follow-up $(t(1,629)=-3.00 ; P=0.002$; $d=0.15$; $95 \% \mathrm{CI}, 0.05,0.25)$, and increases in perceived agency at post-intervention $\left(t(1,629)=-3.22 ; \quad P_{\text {adj }}<0.001 ; d=-0.15\right.$; $95 \% \mathrm{CI},-0.24,-0.05)$ and follow-up $(t(1,629)=2.46 ; P=0.01$; $d=-0.12 ; 95 \% \mathrm{CI},-0.22,-0.02)$, versus control-group adolescents. Improvements in hopelessness at post-intervention and follow-up 
Table 3 | Means and standard deviations for the primary and secondary study outcomes at baseline, post-intervention (where applicable) and three-month follow-up

\begin{tabular}{llll}
$\begin{array}{l}\text { Outcome by } \\
\text { treatment } \\
\text { assignment }\end{array}$ & $\begin{array}{l}\text { Baseline, } \\
\text { mean (s.d.) }\end{array}$ & $\begin{array}{l}\text { Post-intervention, } \\
\text { mean (s.d.) }\end{array}$ & $\begin{array}{l}\text { Three-month } \\
\text { follow-up, mean } \\
\text { (s.d.) }\end{array}$ \\
\hline
\end{tabular}

\begin{tabular}{|c|c|c|c|}
\hline \multicolumn{4}{|l|}{ Placebo control } \\
\hline $\begin{array}{l}\text { Beck } \\
\text { Hopelessness } \\
\text { Scale-four-item } \\
\text { version }\end{array}$ & $1.71(0.76)$ & $1.36(0.75)$ & $1.51(0.82)$ \\
\hline $\begin{array}{l}\text { State Hope } \\
\text { Scale-Agency } \\
\text { Subscale }\end{array}$ & $3.88(1.39)$ & $5.26(1.52)$ & $4.89(1.64)$ \\
\hline CDI-SF & $14.31(4.12)$ & - & $12.57(4.97)$ \\
\hline $\begin{array}{l}\text { COVID-19 Trauma } \\
\text { Symptoms }\end{array}$ & $2.80(0.65)$ & - & $2.83(0.63)$ \\
\hline $\begin{array}{l}\text { Generalized } \\
\text { Anxiety Disorder-7 }\end{array}$ & $3.01(0.73)$ & - & $2.78(0.79)$ \\
\hline $\begin{array}{l}\text { Dietary } \\
\text { Restriction } \\
\text { Screener }\end{array}$ & $0.66(0.43)$ & & $0.63(0.43)$ \\
\hline \multicolumn{4}{|l|}{ Project ABC (BA-SSI) } \\
\hline $\begin{array}{l}\text { Beck } \\
\text { Hopelessness } \\
\text { Scale-four-item } \\
\text { version }\end{array}$ & $1.72(0.77)$ & $1.22(0.73)$ & $1.37(0.80)$ \\
\hline $\begin{array}{l}\text { State Hope } \\
\text { Scale-Agency } \\
\text { Subscale }\end{array}$ & $3.93(1.40)$ & $5.68(1.43)$ & $5.06(1.63)$ \\
\hline CDI-SF & $14.15(4.06)$ & - & $11.47(5.04)$ \\
\hline $\begin{array}{l}\text { COVID-19 Trauma } \\
\text { Symptoms }\end{array}$ & $2.75(0.66)$ & - & $2.73(0.64)$ \\
\hline $\begin{array}{l}\text { Generalized } \\
\text { Anxiety Disorder-7 }\end{array}$ & $2.96(0.76)$ & - & $2.73(0.82)$ \\
\hline $\begin{array}{l}\text { Dietary } \\
\text { Restriction } \\
\text { Screener }\end{array}$ & $0.69(0.41)$ & & $0.57(0.43)$ \\
\hline \multicolumn{4}{|c|}{ Project Personality (GM-SSI) } \\
\hline $\begin{array}{l}\text { Beck } \\
\text { Hopelessness } \\
\text { Scale-four-item } \\
\text { version }\end{array}$ & $1.72(0.76)$ & $1.21(0.72)$ & $1.39(0.83)$ \\
\hline $\begin{array}{l}\text { State Hope } \\
\text { Scale-Agency } \\
\text { Subscale }\end{array}$ & $3.96(1.44)$ & $5.50(1.48)$ & $5.17(1.65)$ \\
\hline CDI-SF & $14.22(4.13)$ & - & $11.58(5.08)$ \\
\hline $\begin{array}{l}\text { COVID-19 Trauma } \\
\text { Symptoms }\end{array}$ & $2.82(0.64)$ & - & $2.76(0.66)$ \\
\hline $\begin{array}{l}\text { Generalized } \\
\text { Anxiety Disorder-7 }\end{array}$ & $3.00(0.74)$ & - & $2.68(0.81)$ \\
\hline $\begin{array}{l}\text { Dietary Restriction } \\
\text { Screener }\end{array}$ & $0.68(0.42)$ & & $0.60(0.43)$ \\
\hline
\end{tabular}

did not significantly differ for adolescents assigned to the BA-SSI relative to adolescents assigned to the GM-SSI (at post-intervention, $t(1,632)=0.50 ; \quad P=0.497 ; d=-0.03 ; 95 \% \mathrm{CI},-0.06,0.13$; at follow-up, $t(1,632)=-0.55 ; P=0.585 ; d=0.03 ; 95 \% \mathrm{CI},-0.12$, 0.07). However, adolescents assigned to the BA-SSI reported larger increases in perceived agency, relative to adolescents assigned to the
GM-SSI, at post-intervention $\left(t(1,632)=3.22 ; P_{\text {adj }}=0.001 ; d=0.16\right.$; 95\% CI, 0.06, 0.26). This difference did not persist at follow-up $(t(1,632)=-1.04 ; P=0.30 ; d=0.05 ; 95 \% \mathrm{CI},-0.15,0.05)$.

COVID-19-related trauma and anxiety outcomes. We investigated COVID-19-related trauma and anxiety as pre-registered secondary outcomes (see Supplementary Tables 2 and 3 for the full results of the regression analyses, for which all assumptions for interpretation were met). Compared with adolescents randomized to the control SSI, adolescents randomized to the BA-SSI did not report decreases in generalized anxiety $(t(1,637)=-0.37 ; P=0.72$; $d=0.02$; $95 \% \mathrm{CI},-0.08,0.12)$ or COVID-19-related trauma symptoms $(t(1,637)=-1.94 ; P=0.05 ; d=0.10 ; 95 \% \mathrm{CI},-0.001,0.19)$ from baseline to three-month follow-up. However, adolescents randomized to the GM-SSI did report significant decreases in generalized anxiety symptom severity $(t(1,629)=-2.08 ; P=0.038$; $d=0.10 ; 95 \%$ CI, 0.006, 0.20) and COVID-19-related trauma symptoms $(t(1,629)=-2.08 ; P=0.037 ; d=0.10 ; 95 \% \mathrm{CI}, 0.006,0.20)$ at three-month follow-up. Adolescents assigned to the BA-SSI and GM-SSI did not differ in their COVID-19-related trauma symptom reductions from pre-intervention to three-month follow-up $(t(1,632)=0.28 ; P=0.78 ; d=-0.01 ; 95 \% \mathrm{CI},-0.008,0.11)$; however, the GM-SSI led to greater reductions in generalized anxiety symptom severity than the BA-SSI $(t(1,632)=2.01 ; P=0.044 ; d=-0.10$; $95 \%$ CI, 0.002, 0.20).

Restrictive eating outcomes. Restrictive eating was investigated as a post hoc, exploratory secondary outcome (see Supplementary Table 8 for the full results of the regression analysis, for which all assumptions for interpretation were met). Compared with the control-group adolescents, those who received the BA-SSI reported significant reductions in past-month restrictive eating from pre-intervention to three-month follow-up $(t(1,637)=3.07$; $P=0.002 ; d=0.15 ; 95 \% \mathrm{CI}, 0.06,0.25)$. Likewise, adolescents randomized to the GM-SSI reported significant reductions in restrictive eating from pre-intervention to three-month follow-up $(t(1,629)=1.99 ; P=0.047 ; d=0.10 ; 95 \%$ CI, 0.001 , $0.20)$. Adolescents randomly assigned to the BA-SSI and GM-SSI did not significantly differ in restrictive eating changes from pre-intervention to three-month follow-up $(t(1,632)=-1.31$; $P=0.153 ; d=-0.07 ; 95 \% \mathrm{CI},-0.16,0.03)$.

Sensitivity analyses. To test the robustness of our results, we ran two sets of additional analyses using alternative approaches to handling missing data for all pre-registered analyses. Both of these were completers-only analyses; one used listwise deletion for participants lacking follow-up data, and the other used multiple imputation to estimate follow-up outcomes for all participants but excluded participants who did not complete their assigned SSI. The code and results from these analyses are available on the Open Science Framework (https://osf.io/8mk6x/). Compared with the control SSI, the overall effects of the GM-SSI and BA-SSI on depressive symptoms, generalized anxiety symptoms, post-intervention and three-month hopelessness, post-intervention and three-month perceived agency, and three-month generalized anxiety symptoms were generally unchanged using alternative missing data approaches. Only a few minor differences emerged from pre-registered imputation analyses, exclusively with respect to secondary outcomes. In our completers-only analyses with listwise deletion, the effect of the GM-SSI on COVID-19-related trauma symptoms at three-month follow-up was non-significant, versus the control $(t(987)=1.81 ; P=0.07 ; d=0.12 ; 95 \% \mathrm{CI},-0.01,0.12)$; also, the GM-SSI showed a significantly stronger (more positive) effect on generalized anxiety symptoms at three-month follow-up than did the BA-SSI $(t(994)=2.00 ; P=0.045 ; d=0.13 ; 95 \%$ CI, $0.002,0.25)$. In our completers-only analyses with imputation for follow-up 


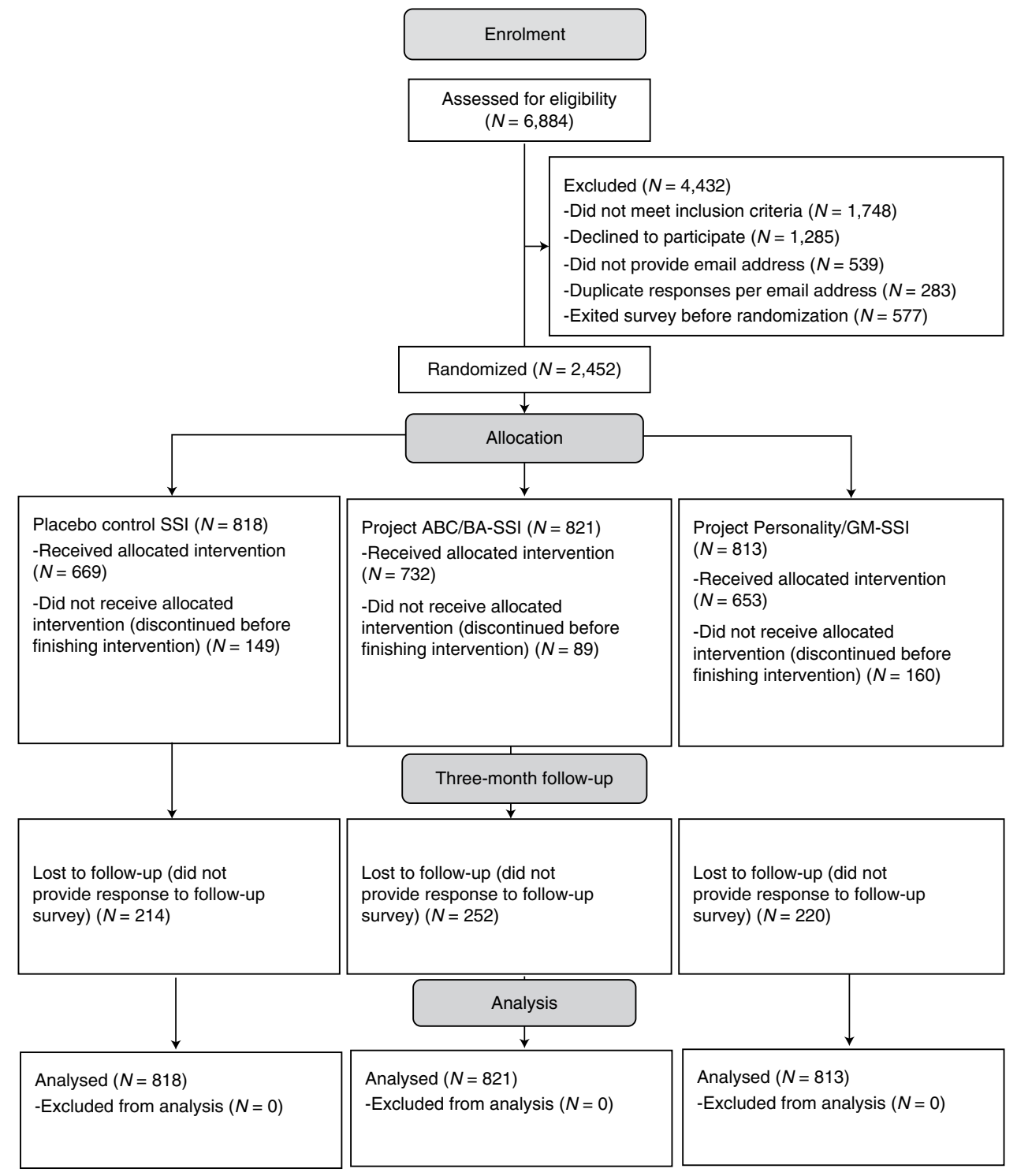

Fig. 2 | CONSORT diagram. CONSORT diagram displaying participant flow through study screening, randomization, intervention completion and follow-up assessment completion.

non-completers, the BA-SSI significantly reduced COVID19-related trauma symptoms at three-month follow-up, versus the control $(t(1,637)=2.51 ; P=0.01 ; d=0.12 ; 95 \% \mathrm{CI}, 0.03,0.22)$, and the GM-SSI had a significantly greater, positive effect on generalized anxiety symptoms, versus the BA-SSI $(t(1,632)=2.20 ; P=0.02$; $d=0.11 ; 95 \% \mathrm{CI}, 0.01,0.21)$. Thus, the overall results patterns were similar-showing only minor differences with respect to secondary outcomes-regardless of our approach to handling missing data.

\section{Discussion}

In a nationwide randomized trial, we tested whether two half-hour, self-administered SSIs teaching BA and GM, respectively, reduced hopelessness, improved perceived agency and lessened symptoms of depression, anxiety and COVID-19-related trauma in high-symptom adolescents. Compared with a supportive control SSI, both active interventions led to significantly greater improvements in depressive symptoms from baseline to three-month follow-up (BA-SSI $d=0.18$; GM-SSI $d=0.18$ ). Additionally, both active interventions led to greater decreases in hopelessness at post-intervention (BA-SSI $d=0.26$; GM-SSI $d=0.28$ ) and follow-up (BA-SSI $d=0.17$; GM-SSI $d=0.16$ ) and increases in perceived agency at post-intervention (BA-SSI $d=-0.31$; GM-SSI $d=-0.15$ ), relative to the control; the GM-SSI also led to increases in perceived agency at follow-up $(d=0.17)$. Regarding differential intervention effects, the GM-SSI (but not the BA-SSI) reduced three-month generalized anxiety symptoms $(d=0.10)$ and COVID-19-related trauma symptoms $(d=0.10)$ relative to the control. The BA-SSI led to greater post-SSI increases in perceived agency than did the GM-SSI $(d=0.16)$, whereas the GM-SSI led to greater three-month reductions in generalized anxiety symptoms than did the BA-SSI $(d=0.10)$. Furthermore, exploratory post hoc analyses revealed that both the BA-SSI and the GM-SSI significantly reduced restrictive eating from pre-intervention to three-month follow-up, compared with the control. Sensitivity analyses using two alternative approaches to handling missing data yielded similar patterns of results, lending confidence to the observed outcomes for the pre-registered tests. Indeed, the effect sizes observed for the BA-SSI's and the GM-SSI's effects on depressive symptoms were identical to meta-analytic estimates of SSIs' effects on youth depressive symptoms (for example, Schleider and colleagues $^{17}$ identified a meta-analytic effect on depressive symptoms of $d=0.18$ ). 
Both active SSIs yielded modest reductions, on average, in adolescent depressive symptoms. The effect sizes were small, reflecting mean three-month reductions of 2.64 points on the CDI-SF. Nonetheless, for several reasons, the results hold clinical and practical importance. First, before and especially during the COVID-19 pandemic, adolescents experiencing depression have remained unlikely to access any mental health treatment-let alone evidence-based support-due to structural and logistical barriers. Many of these barriers, such as poverty, proximity to providers and insurance status, are impossible to modify without large-scale policy intervention. Thus, even modest overall clinical improvements produced by cost-free, 20 - to 30-minute, self-guided interventions suggest their promise to enable efficient clinical benefits, which could be multiplied at the population level. Moving forwards, research might focus less on how to strengthen the average magnitude of these SSIs' impacts and more on identifying subsets of best-responder adolescents and guiding tailored dissemination based on individual odds of benefit.

Second, this trial represents a confirmatory extension of prior work suggesting the promise of SSIs for adolescent mental health. The use of an active control (which itself produced significant within-group decreases in depression symptoms), a three-month follow-up, a large sample and high reproducibility (due to pre-registration and open-access intervention materials, data and analytic code) strengthen confidence in our results. Indeed, the effects of many SSIs fall to near zero after multi-month follow-up periods, and the effects of most adolescent mental health treatments decrease substantially when tested against active (versus no-treatment) controls ${ }^{13,17}$. Furthermore, the average number of participants in randomized trials of youth psychotherapies to date has been 68.69, rendering many trials underpowered for robust effectiveness tests ${ }^{13}$. This study is among the largest US-based randomized controlled trials of any psychosocial treatment for adolescent depression to date.

Third, this study supports acceptability and effectiveness for brief, self-guided depression interventions among highly diverse adolescents. The sample included population-congruent representation of Black, Hispanic, Asian and Native American youth in the United States; the participants included youths living in all 50 US states; and $80 \%$ of the participants identified as sexual minorities. Although sexual minority youths were arguably over-represented in this study, most youth psychotherapy trials routinely include samples that are $>90 \%$ White and seldom assess sexual orientation ${ }^{13}$. This sample's diversity thus extends the youth mental health knowledge base, filling gaps in the youth intervention literature that have long remained unaddressed.

Fourth, the positive effects on depressive symptoms are notable because (1) both active interventions yielded significant benefits, even in the high-stress context of a global pandemic; and (2) the results address the need for more potent adolescent depression interventions. In fact, the overall effect sizes across all trials of youth depression treatments have decreased from 1960 to the present ${ }^{29}$. The SSIs in this trial might help improve population-level youth depression symptoms and outcomes, if disseminated broadly.

Both active SSIs yielded improvements in post-intervention hopelessness and perceived agency, although the BA-SSI increased agency more than the GM-SSI. These results align with evidence that many psychotherapy change mechanisms may be more similar than different across programmes, even when those programmes are thought to target distinct mechanisms ${ }^{30}$. Furthermore, both SSIs are designed per a common set of components ${ }^{20}$ including neuroscience-based psychoeducation, narratives from peers and opportunities to provide advice to others. These shared elements may promote a common set of adaptive thinking styles-perhaps reflected by the SSIs' equivalent impacts on hopelessness. By contrast, the BA-SSI's larger impacts on short-term perceived agency may reflect its unique elements, such as creating a personalized action plan for engaging in valued activities. Creating this plan may strengthen adolescents' confidence in their ability to solve future mood-related problems, at least in the short term. Indeed, items included in this study's assessment of agency (for example, "There are lots of ways around problems I am facing right now") map more directly onto the BA-SSI than the GM-SSI.

Notably, according to the pre-registered analyses, the GM-SSI reduced three-month generalized anxiety and COVID-19-related trauma versus the control, but the BA-SSI did not. This pattern fits with the intended content specificity (or lack thereof) of each SSI. The BA-SSI provides psychoeducation specific to depression and low mood, whereas the GM-SSI is intentionally not diagnosis-specific; its message is that everyone has potential for change across multiple domains, including abilities to cope with anxiety and depression. Prior studies testing the GM-SSI in high-symptom adolescents have also found programme benefits for both depression and anxiety symptoms ${ }^{19}$. Future work may test the prospect of matching youth to the BA-SSI or GM-SSI on the basis of individual clinical needs and the strengths of each intervention.

The post hoc exploratory analyses revealed that both the GM-SSI and the BA-SSI significantly reduced restrictive eating behaviours at three-month follow-up, relative to the control. This result was unanticipated but aligned with research suggesting close ties between mood problems and disordered eating ${ }^{31}$, with interventions targeting restrictive eating often yielding improvements in $\operatorname{mood}^{28}$. However, few youth-depression-focused intervention trials have evaluated restrictive eating as a secondary outcome. Our results support the utility of including such outcomes in depression treatment studies, including trials of SSIs. Given the dearth of treatment resources available to youths experiencing eating disorder symptoms $\mathrm{s}^{32}$ and the inherent accessibility of the interventions in this trial, the potential for SSIs to directly mitigate restrictive eating merits future study.

How might these SSIs fit into broader youth mental-health-care ecosystems? In considering possibilities, it is crucial to note that SSIs are not panaceas. The overall effects were modest, some adolescents will still require longer-term services, and policy changes remain needed to improve youth access to higher-intensity treatments in addition to brief, immediately accessible resources. Nonetheless, the SSIs in this trial may offer rapidly scalable, evidence-based support for all youths with depressive symptoms-many of whom would otherwise access no support at all. Given the brevity and flexibility of these programmes, they may be offered within diverse youth care systems (for example, primary care, emergency rooms and schools), complementing existing support structures while retaining the potential to benefit youths unable to access further care.

A notable feature of this study is adolescents' ability to self-refer into SSIs. In many US states, adolescents aged $\geq 12$ may consent, without a parent or guardian, to mental and physical health care. These laws were enacted to increase adolescents' likelihood of seeking and obtaining care. For myriad reasons, allowing youths to participate in minimal-risk online SSIs without requiring parental consent may be a critical path towards increasing youth treatment access and use. This study suggests that adolescents can safely participate in online SSIs and that SSIs may reduce both short-term and longer-term mental health difficulties. Requiring parent or guardian approval for adolescents to try these online activities could prevent thousands of youth from receiving minimal-risk, free, evidence-based support $^{33,34}$. Unfortunately, requiring parent permission may pose disproportionate barriers for sexual minority, gender minority and racial/ethnic minority youth, for whom concerns about caregiver stigma or dismissal (linked to the rejection of one's identity, beliefs about seeking mental health treatment or both) often prevent youth from disclosing treatment needs to family ${ }^{35}$. Although not all states have adopted these guidelines, the SSIs tested here pose minimal 
risk to safety and may offer a common-sense path to increasing access to mental health support among adolescents.

Some study limitations warrant consideration and suggest directions for future work. First, the eligibility screening relied on adolescents' interest in completing an online study and required Internet access. Some adolescents may require support from adults to access mental health treatment, some may lack Internet access, others may not have the time for this type of study and others still might speak languages other than English (the SSIs were not available in other languages at the time of this trial). The findings therefore may not apply uniformly across adolescents. However, most prior trials of psychosocial interventions for adolescents have required parent referral and consent; by eliminating this barrier, this study may have included adolescents under-represented in prior clinical trials, such as adolescents who are not open with caregivers about mental health difficulties.

Second, attrition at follow-up was $\sim 40 \%$. Missing data were addressed via a rigorous approach that has shown utility with higher missing data rates than observed here, and sensitivity analyses using alternative data approaches yielded similar patterns of results $^{36}$. Although the results may have been influenced by these missing data, this rate is comparable to those observed in previous randomized trials of youth depression interventions (for example, the Treatment for Adolescents with Depression Study) ${ }^{7,37}$. Future investigations might formally compare methods for increasing participant retention across longer-term study periods.

Third, some groups of youth were over-represented in our sample (for example, sexual minority youth), whereas others were underrepresented (for example, boys). These sample characteristics are unlikely to reflect our Instagram-based recruitment approach, as $72 \%$ of teens ages 13-17 actively use Instagram, nearly half of whom are boys ${ }^{38}$. Our sample may thus reflect youths most readily drawn to taking part in online self-help activities. Focus-group-based and mixed-methods research, geared towards gathering youth feedback and guidance, may forward efforts to engage adolescent boys in digital, mental-health-focused SSIs.

Fourth, although SSI type did not predict attrition at three-month follow-up, youths randomized to the BA-SSI were more likely than those in the GM-SSI and control conditions to complete their assigned intervention (although the completion rates were very high across conditions ( $>80 \%$ ) compared with the majority of self-guided digital mental health programmes ${ }^{27}$ ). It is possible that the greater interactivity of the BA-SSI (for example, the creation of an action plan) contributed to this higher completion rate, but tests of engagement-enhancing components of online SSIs remain a key direction for future work.

Lastly, we did not formally assess youths' use of other mental health supports during the study period. Notably, SSIs may function best as complements to (rather than replacements for) other forms of care. Indeed, a prior randomized trial demonstrated no effect of external mental health treatment (for example, psychotherapy and/ or medication) on response to the GM-SSI, versus a placebo control, across a nine-month follow-up ${ }^{19}$. Future trials might collect data to capture the role of SSIs in the context of youths' full range of formal and informal mental health supports.

In sum, our results suggest that free-of-charge, 20- to 30-minute online interventions can reduce depressive symptoms, hopelessness and anxiety and strengthen perceived agency across three months in high-symptom adolescents, even in the context of a global pandemic. Although differences across the active interventions emerged, these brief supports could be made freely available for adolescents to complete anytime, from any location, regardless of traditional barriers to mental health care.

\section{Methods}

Ethics information. The procedures were approved by the University of Denver institutional review board, and adolescents provided online assent before participating. To maintain adolescents' confidentiality and minimize access barriers (for example, discomfort disclosing psychological distress, as parents are often unaware of their adolescents' depressive symptoms, including suicidal ideation, in up to $80 \%$ of cases $)^{39}$, parent permission was not required to participate in this study (waived by the university institutional review board). All procedures were pre-registered prospectively, before the enrolment of the first participant (ClinicalTrials.gov: NCT04634903). As this was a minimal-risk study (per the institutional review board's determination), we did not expect any adverse events to occur during the study period; as such, we included no explicit assessments of adverse events. No incidental adverse events of any kind were reported by the participants or identified by the researchers during the study period.

Recruitment and resulting sample. The participants learned about the study through Instagram advertisements following established ethics guidelines for passive, social-media-based recruitment ${ }^{40}$. The posts included invitations to determine eligibility for a confidential, online psychology study, for which participants could earn up to US\$20 in gift cards; Supplementary Fig. 1 depicts an example advertisement from our study. Instagram posts linked to a Qualtrics survey; the first page included the study details and an invitation to complete an eligibility screener. The inclusion criteria were (1) being between 13 and 16 years old (inclusive); (2) comfort reading and writing in English; (3) Internet and computer, laptop or smartphone access; and (4) endorsement of elevated depressive symptoms, per a score of $\geq 2$ on the Patient Health Questionnaire- 2 (this cut-off prioritizes sensitivity over specificity in identifying youth with elevated depressive symptoms $)^{41}$. Eligible adolescents then reviewed an online assent form inviting them to participate. Recruitment took place from 19 November to 6 December 2020 (on which date $>2,400$ youth had been randomized to an intervention condition). Three-month follow-up surveys were completed by 15 March 2021 (youth received three-week windows to finish their follow-up survey). Of the 6,884 youths who completed the screener, 3,851 were eligible and agreed to participate; 2,452 completed the baseline survey and were randomized to an SSI; and 1,766 of those randomized were retained at follow-up. The rates of follow-up survey completion did not differ by intervention condition The participants could complete the study from any US location (Supplementary Fig. 2 illustrates the geographic distribution of the study participants), using any Internet-equipped device.

Procedures. Per the CONSORT diagram (Fig. 1), the participants were invited to complete pre-SSI questionnaires, one of three 20-30 minute SSIs (youths were randomly assigned to one of three SSIs in a 1:1:1 ratio per a Qualtrics-embedded randomizer; youth and investigators were masked to condition assignment until after all data collection was complete), and post-SSI questionnaires within one 50- to 60-minute session. Youths then received an email invitation three months later to complete a 10-minute follow-up questionnaire (also via Qualtrics), including a subset of measures from the baseline survey, to assess the SSIs' effects on the primary and secondary outcomes. After all data collection was complete, the researchers learned each youth's condition assignment, and both active interventions were offered to all participants. Additionally, the participants received a resource list of hotlines, textlines and online psychoeducational resources to facilitate engagement with additional mental health supports, if desired. Adolescents were also invited to contact the research team at any time during the study with questions or for further support in accessing mental health support beyond the study's scope.

Interventions. The BA-SSI (ABC Project $\left.{ }^{20}\right)$ includes five elements: (1) an introduction to the programme's rationale-that engaging in values-based activities that build pleasure and accomplishment can combat sad mood and low self-esteem; (2) psychoeducation about depression, including how behaviour shapes feelings and thoughts; (3) a life values assessment, where youth identify key areas (family relationships, friendships, school or hobbies) from which they draw (or once drew) enjoyment and meaning; (4) the creation of an activity action plan, where youth identify (from pre-generated lists) and personalize (in guided exercises) three activities to target for change; and (5) an exercise in which youths write about benefits that might result from engaging in each activity, an obstacle that might keep them from doing the activities and a strategy for overcoming the identified obstacles. The intervention materials are available at https://osf.io/ch2tg/.

The GM-SSI (Project Personality ${ }^{18,19}$ ) is a self-administered youth programme that includes five elements: (1) an introduction to the brain and a lesson on neuroplasticity; (2) testimonials from older youths who describe their views that traits are malleable, due to the brain's plasticity; (3) further stories by older youths, describing times when they used 'growth mindsets' to persevere during social or emotional setbacks; (4) study summaries noting how and why personality can change; and (5) an exercise in which youths write notes to younger students, using scientific information to explain people's capacity for change. The intervention materials are available at https://osf.io/a9uv2/.

The Supportive Therapy SSI (placebo) ${ }^{18,19}$ is self-administered and structurally similar to the other SSIs (for example, it is length-matched, includes peer narratives and contains the same number of writing activities), but it is designed to control for non-specific aspects of completing a generally supportive online activity. 
The programme encourages participants to express emotions to close others; it does not teach specific skills. In a previous trial, this programme predicted smaller reductions in youth internalizing problems versus a GM-SSI ${ }^{19}$. The intervention materials are available at https://osf.io/u4axs/.

Per prior open and randomized trials including the ABC Project, Project Personality and the Supportive Therapy SSI, each of these interventions takes approximately $20-30$ minutes for adolescents experiencing depressive symptoms to complete $^{19,20}$

Outcomes. Listed below are the key primary and secondary trial outcomes for which we pre-registered confirmatory (three-month depressive symptoms, post-intervention hopelessness and post-intervention perceived agency) and exploratory analyses (three-month hopelessness, three-month perceived agency, three-month generalized anxiety and three-month COVID-19-related trauma). We also included a handful of additional exploratory secondary outcomes, as noted in our clinicaltrials.gov registration. These were three-month restrictive eating, presence of lifetime suicidal ideation, three-month approach-based coping and post-intervention implicit theory of personality. Because no analyses were pre-registered regarding these outcomes, the results of post hoc analyses testing intervention effects on these variables are detailed in Supplementary Tables 8-11. Additionally, we include results for one of these variables (restrictive eating) in the main text due to unanticipated, significant intervention effects revealed in post hoc analyses. Lastly, in Supplementary Table 12, we list all measures collected as part of the trial, defined as primary, secondary or 'other', as well as the time points at which they were administered to the participants (including baseline-only, exploratory 'other' measures for which no analyses were pre-registered and no descriptive statistics are reported in this manuscript).

Adolescent depressive symptoms (primary outcome). Depressive symptom severity was assessed using the CDI-SF, a reliable, valid measure of youth depression severity ${ }^{42}$. Twelve items are scored from 0 to 2 , with higher summed scores reflecting greater overall depression symptom severity. Changes in CDI-SF scores from pre-SSI to three-month follow-up were the primary study outcome. Alphas were 0.77 and 0.85 at baseline and follow-up.

Adolescent anxiety symptoms. Generalized anxiety symptom severity was measured using the Generalized Anxiety Disorder 7, which includes seven items asking respondents how often, during the last two weeks, they were bothered by various anxiety symptoms ${ }^{43}$. The items are rated on a $0-3$ scale and averaged to yield an overall score. Changes in Generalized Anxiety Disorder 7 scores from pre-SSI to follow-up were a secondary outcome. Alphas were 0.89 and 0.90 at baseline and follow-up.

COVID-19-related trauma symptoms. The Child Trauma Screen-Reaction Scale (CTS-RS) was administered at pre-SSI and three-month follow-up ${ }^{44}$. The CTS-RS is a reliable, valid self-report measure of traumatic stress symptom severity, including event-related somatic symptoms, intrusive memories, avoidance, sleep problems, and mood and behavioural changes. The instructions read: "For many, the COVID-19 (or 'coronavirus') pandemic has been scary or very upsetting. Sometimes, events that are scary or upsetting can affect how people think, feel, and act. The next questions ask how you have been feeling and thinking recently.' Youth rated the frequencies of six types of traumatic stress symptoms, per past-month frequency, on the basis of a 0-3 scale; the ratings were averaged to yield an overall severity score. Changes in CTS-RS scores from pre-SSI to follow-up were a secondary outcome. Alpha was 0.73 at both baseline and follow-up.

Perceived agency. The Agency Subscale of the State Hope Scale is a reliable, valid three-item self-report measure of youths' perceived ability to generate plans and work towards their goals $s^{45}$. Pre- and post-intervention, youths rated their agreement with three statements reflecting how they felt about themselves "right now" on an eight-point scale. Alphas were $0.82,0.83$ and 0.80 at pre-SSI, post-SSI and follow-up.

Hopelessness. The Beck Hopelessness Scale- 4 is a reliable, shortened version of the 20 -item scale used to measure hopelessness in youth ${ }^{46,47}$. Pre- and post-intervention and at follow-up, the participants rated their agreement with four statements reflecting their sense of hopelessness "right now, in this moment" on a 0-3 Likert scale. Alphas were $0.84,0.87$ and 0.88 at pre-SSI, post-SSI and follow-up.

Intervention acceptability. The PFS asks youth to rate agreement with seven statements indicating the perceived acceptability of an SSI (for example, "I enjoyed the program") on a five-point Likert scale ( $1=$ "really disagree"; $5=$ "totally agree" $)^{20,48}$. We pre-registered that scores of $\geq 3.5 / 5$ on any given PFS item would reflect an 'acceptable' rating on that item.

Restrictive (disordered) eating. Restrictive eating behaviours were measured at pre-intervention and three-month follow-up using the Dietary Restriction Screener (DRS-2) ${ }^{49}$. The DRS-2 is a two-item measure evaluating restrictive eating behaviours. The two items ask participants whether or not they have engaged in restrictive eating in the past year or in the past three months $(0=$ no; $1=$ yes $)$. The DRS-2 was administered at pre-SSI and three-month follow-up. Given the timing of the assessments in this study, we examined intervention effects on the presence of past-month restrictive eating only. No analyses were pre-registered for this outcome, and all analyses were conducted post hoc with respect to intervention effects on restrictive eating.

Power analysis. The R package pwr $^{50}$ was used to calculate the power for all planned contrasts detailed below. A sample size of $N=2,400$ (800 per group) and two time points (pre-intervention and three-month follow-up) yield $98 \%$ power to detect a small effect size of $d=0.20$ on all outcomes when comparing SSIs. Notably, our final sample $(N=2,452)$ was 52 participants greater than our pre-registered sample size, as a group of youths completed our eligibility screener within hours of a study advertisement gaining traction on Instagram. We stopped recruitment as soon as we learned that our pre-registered threshold had been met.

Analysis plan. We ran a series of linear regressions to test whether the GM-SSI and BA-SSI predicted (1) improvements in proximal post-SSI targets (perceived agency and hopelessness) and (2) reductions in adolescent depressive symptom severity (the primary study outcome) from pre-SSI to three-month follow-up, relative to (1) each other and (2) the control SSI, when considered independently. SSI condition was a categorical predictor in these models, and parallel linear regressions were conducted to assess intervention effects on secondary and other exploratory study outcomes. We imputed participant-level missing data using the expectation-maximization and bootstrapping algorithm implemented with Amelia II in $\mathrm{R}^{51}$, allowing more conservative intent-to-treat analyses than other approaches (for example, listwise deletion and last observation carried forwards). We imputed as many datasets as there were percentage points of missing data for an outcome, rounding to the next-highest percentage (for example, if $5.4 \%$ of data was missing on an outcome, we created 6 imputed datasets), allowing us to retain high power despite missing data. Cohen's $d$ and 95\% CIs for the analyses were calculated using $t$ values for treatment effects obtained from analyses with the MOTE package in $\mathrm{R}^{52}$. The false discovery rate (FDR) was applied to identify potential false-positive results $^{53}$. The results from the pre-registered tests described above were considered significant if FDR-corrected $P<0.05$, and all tests were two-tailed. Lastly, for exploratory purposes (and to contextualize within-group changes in symptom levels over time in each condition), we computed effect sizes reflecting within-group intervention effects on depression (the primary outcome) for each SSI condition.

Differential SSI completion and attrition. We ran post hoc $Z$-tests of proportion analyses to assess whether the rates of SSI completion and three-month follow-up survey completion differed by condition assignment. We then ran two separate logistic regression models with baseline depression symptom severity, age, gender identity, sexual orientation, romantic/sexual attraction and racial identity to determine whether those characteristics could predict which participants would drop out either before completing the intervention or before initiating the three-month follow-up.

Sensitivity analyses. Given the high rate of missing data that characterizes many clinical trials, and to gauge whether the results might have differed due to our approach to handling missing data, we re-ran the pre-registered analyses using two alternative approaches to handling missing data. First, we ran a completers-only analysis using listwise deletion for participants who did not complete the follow-up survey. Second, we ran a completers-only analysis wherein participants who did not complete their assigned SSI were excluded, with multiple imputation (as described above) for three-month follow-up outcomes.

Reporting Summary. Further information on research design is available in the Nature Research Reporting Summary linked to this article.

\section{Data availability}

Anonymized participant-level data are available on the Open Science Framework: https://doi.org/10.17605/OSF.IO/8MK6X.

\section{Code availability}

The analytic code is available on the Open Science Framework: https://doi.org/ 10.17605/OSF.IO/8MK6X.

Received: 8 April 2021; Accepted: 18 October 2021; Published online: 9 December 2021

\section{References}

1. Golberstein, E., Wen, H. \& Miller, B. F. Coronavirus disease 2019 (COVID-19) and mental health for children and adolescents. JAMA Pediatr. 174, 819-820 (2020)

2. Whiteford, H. A. et al. Global burden of disease attributable to mental and substance use disorders: findings from the Global Burden of Disease Study 2010. Lancet 382, 1575-1586 (2013). 
3. Hammen, C., Brennan, P. A. \& Keenan-Miller, D. Patterns of adolescent depression to age 20: the role of maternal depression and youth interpersonal dysfunction. J. Abnorm. Child Psychol. 36, 1189-1198 (2008).

4. Wilson, S., Hicks, B. M., Foster, K. T., McGue, M. \& Iacono, W. G. Age of onset and course of major depressive disorder: associations with psychosocial functioning outcomes in adulthood. Psychol. Med. 45, 505-514 (2015).

5. Forman-Hoffman, V. et al. Screening for Major Depressive Disorder in Children and Adolescents: A Systematic Review for the U.S. Preventive Services Task Force Evidence Synthesis No. 116, AHRQ Publication No. 13-05192-EF-1 (Agency for Healthcare Research and Quality, 2016).

6. Avenevoli, S., Swendsen, J., He, J. P., Burstein, M. \& Merikangas, K. R. Major depression in the National Comorbidity Survey-Adolescent Supplement: prevalence, correlates, and treatment. J. Am. Acad. Child Adolesc. Psychiatry 54, 37-44 (2015)

7. March, J. S. et al. The Treatment for Adolescents with Depression Study (TADS): long-term effectiveness and safety outcomes. Arch. Gen. Psychiatry 64, 1132-1144 (2007).

8. Vitiello, B. et al. Long-term outcome of adolescent depression initially resistant to SSRI treatment. J. Clin. Psychiatry 72, 388-396 (2011).

9. Fried, E. I. \& Nesse, R. M. Depression sum-scores don't add up: why analyzing specific depression symptoms is essential. BMC Med. 13, 72 (2015)

10. Fried, E. I. \& Nesse, R. M. Depression is not a consistent syndrome: an investigation of unique symptom patterns in the STAR ${ }^{*} \mathrm{D}$ study. J. Affect. Disord. 172, 96-102 (2015).

11. Hollon, S., Garber, J. \& Shelton, R. Treatment of depression in adolescents with cognitive behavior therapy and medications: a commentary on the TADS project. Cogn. Behav. Pract. 12, 149-155 (2005).

12. Chu, B. C., Colognori, D., Weissman, A. S. \& Bannon, K. An initial description and pilot of group behavioral activation therapy for anxious and depressed youth. Cogn. Behav. Pract. 16, 408-419 (2009).

13. Weisz, J. R. et al. What five decades of research tells us about the effects of youth psychological therapy: a multilevel meta-analysis and implications for science and practice. Am. Psychol. 72, 79-117 (2017).

14. Kataoka, S. H., Zhang, L. \& Wells, K. B. Unmet need for mental health care among U.S. children: variation by ethnicity and insurance status. Am. J. Psychiatry 159, 1548-1555 (2002).

15. Harpaz-Rotem, I., Leslie, D. \& Rosenheck, R. A. Treatment retention among children entering a new episode of mental health care. Psychiatr. Serv. 55, 1022-1028 (2004).

16. Schleider, J. L., Dobias, M. L., Sung, J. Y. \& Mullarkey, M. C. Future directions in single-session youth mental health interventions. J. Clin. Child Adolesc. Psychol. 2, 264-278 (2020).

17. Schleider, J. L. \& Weisz, J. R. Little treatments, promising effects? Meta-analysis of single session interventions for youth psychiatric problems. J. Am. Acad. Child Adolesc. Psychiatry 56, 107-115 (2017).

18. Schleider, J. L., Burnette, J. L., Widman, L., Hoyt, C. \& Prinstein, M. J. Randomized trial of a single-session growth mindset intervention for rural adolescents' internalizing and externalizing problems. J. Clin. Child Adolesc. Psychol. 49, 660-672 (2020).

19. Schleider, J. \& Weisz, J. A single-session growth mindset intervention for adolescent anxiety and depression: nine-month outcomes of a randomized trial. J. Child Psychol. Psychiatry 59, 160-170 (2018).

20. Schleider, J. L., Dobias, M. L., Sung, J. Y., Mumper, E. \& Mullarkey, M. C. Acceptability and utility of an open-access, online single-session intervention platform for adolescent mental health. JMIR Ment. Health 7, e20513 (2020).

21. Miu, A. S. \& Yeager, D. S. Preventing symptoms of depression by teaching adolescents that people can change: effects of a brief incremental theory of personality intervention at 9-month follow-up. Clin. Psychol. Sci. 3, 726-743 (2015).

22. Schleider, J. L., Abel, M. \& Weisz, J. R. Do immediate gains predict long-term symptom change? Findings from a randomized trial of a single-session intervention for youth anxiety and depression. Child Psychiatry Hum. Dev. 50 868-881 (2019).

23. Gawrysiak, M., Nicholas, C. R. N. \& Hopko, D. R. Behavioral activation for moderately depressed university students: randomized controlled trial. J. Couns. Psychol. 56, 468-475 (2009).

24. Armento, M. E. A., McNulty, J. K. \& Hopko, D. R. Behavioral activation of religious behaviors (BARB): randomized trial with depressed students. Psychol. Relig. Spiritual. 4, 206-222 (2012).

25. Huguet, A. et al. A systematic review of cognitive behavioral therapy and behavioral activation apps for depression. PLoS ONE 11, e0154248 (2016)

26. Makover, H. B. et al. Mediators of youth anxiety outcomes 3 to 12 years after treatment. J. Anxiety Disord. 70, 102188 (2020).

27. Baumel, A., Muench, F., Edan, S. \& Kane, J. Objective user engagement with mental health apps: systematic search and panel-based usage analysis. J. Med. Internet Res. 21, e14567 (2019).

28. Stice, E., Marti, C. N., Shaw, H. \& Rohde, P. Meta-analytic review of dissonance-based eating disorder prevention programs: intervention, participant, and facilitator features that predict larger effects. Clin. Psychol. Rev. 70, 91-107 (2019).
29. Weisz, J. R. et al. Are psychotherapies for young people growing stronger? Tracking trends over time for youth anxiety, depression, attention-deficit/ hyperactivity disorder, and conduct problems. Perspect. Psychol. Sci. 14, 216-237 (2019).

30. Cuijpers, P., Reijnders, M. \& Huibers, M. J. The role of common factors in psychotherapy outcomes. Annu. Rev. Clin. Psychol. 15, 207-231 (2019).

31. Stice, E. \& Van Ryzin, M. J. A prospective test of the temporal sequencing of risk factor emergence in the dual pathway model of eating disorders. $J$. Abnorm. Psychol. 128, 119-128 (2019).

32. Weissman, R. S. \& Rosselli, F. Reducing the burden of suffering from eating disorders: unmet treatment needs, cost of illness, and the quest for cost-effectiveness. Behav. Res. Ther. 88, 49-64 (2017).

33. Wilson, C. J. \& Deane, F. P. Brief report: need for autonomy and other perceived barriers relating to adolescents' intentions to seek professional mental health care. J. Adolesc. 35, 233-237 (2012).

34. Samargia, L. A., Saewyc, E. M. \& Elliott, B. A. Foregone mental health care and self-reported access barriers among adolescents. J. Sch. Nurs. 22, 17-24 (2006).

35. Brown, A., Rice, S. M., Rickwood, D. J. \& Parker, A. G. Systematic review of barriers and facilitators to accessing and engaging with mental health care among at-risk young people. Asia Pac. Psychiatry 8, 3-22 (2016).

36. Taylor, L. \& Zhou, X. H. Multiple imputation methods for treatment noncompliance and nonresponse in randomized clinical trials. Biometrics $\mathbf{6 5}$ 88-95 (2009).

37. Treatment for Adolescents with Depression Study (TADS) Teamet al. The Treatment for Adolescents with Depression Study (TADS): outcomes over year of naturalistic follow-up. Am. J. Psychiatry 166, 1141-1149 (2009).

38. Teens' Social Media Habits and Experiences, https://www.pewresearch.org/ internet/wp-content/uploads/sites/9/2018/11/PI_2018.11.28_teens-socialmedia_FINAL4.pdf (Pew Research Center, 2018).

39. Smith, D. M. Y., Lipson, S. M., Wang, S. B., \& Fox, K. R. Online methods in adolescent self-injury research: challenges and recommendations. J. Clin. Child Adolesc. Psychol. https://doi.org/10.1080/15374416.2021.1875325 (2021).

40. Gelinas, L. et al. Using social media as a research recruitment tool: ethical issues and recommendations. Am. J. Bioeth. 17, 3-14 (2017).

41. Richardson, L. P. et al. Evaluation of the PHQ-2 as a brief screen for detecting major depression among adolescents. Pediatrics 125, e1097-e1103 (2010).

42. Kovacs, M. Children's Depression Inventory -2 2nd edn (Multi-Health Systems, 2011)

43. Spitzer, R. L., Kroenke, K., Williams, J. B. \& Löwe, B. A brief measure for assessing generalized anxiety disorder: the GAD-7. Arch. Intern. Med. 166, 1092-1097 (2006)

44. Lang, J. M. \& Connell, C. M. Development and validation of a brief trauma screening measure for children: the Child Trauma Screen. Psychol. Trauma 9, 390-398 (2017)

45. Snyder, C. R. et al. Development and validation of the State Hope Scale. J. Personal. Soc. Psychol. 70, 321-335 (1996).

46. Rhoades, H. et al. Homelessness, mental health and suicidality among LGBTQ youth accessing crisis services. Child Psychiatry Hum. Dev. 49, 643-651 (2018)

47. Forintos, D. P., Rózsa, S., Pilling, J. \& Kopp, M. Proposal for a short version of the Beck Hopelessness Scale based on a national representative survey in Hungary. Community Ment. Health J. 49, 822-830 (2013).

48. Schleider, J. L., Mullarkey, M. C. \& Weisz, J. R. Virtual reality and web-based growth mindset interventions for adolescent depression: protocol for a three-arm randomized trial. JMIR Res. Protoc. 8, e13368 (2019).

49. Haynos, A. F. \& Fruzzetti, A. E. Initial evaluation of a single-item screener to assess problematic dietary restriction. Eat. Weight Disord. 20, 405-413 (2015)

50. Champley, S. pwr: basic functions for power analysis ( $\mathrm{R}$ package version 1.3-0), https://cran.r-project.org/web/packages/pwr/index.html (2020).

51. Honaker, J., King, G. \& Blackwell, M. Amelia II: a program for missing data. J. Stat. Softw. 45, 1-47 (2011).

52. Buchanan, E., Gillenwaters, A., Scofield, J., Valentine, K. MOTE: Measure of the effect-package to assist in effect size calculations and their confidence intervals. R package version 1.0.2 http://github.com/doomlab/MOTE (2019).

53. Benjamini, Y. \& Hochberg, Y. Controlling the false discovery rate: a practical and powerful approach to multiple testing. J. R. Stat. Soc. B 57, 289-300 (1995)

\section{Acknowledgements}

This study was supported by the Office of the Director, National Institutes of Health under an 'Emergency COVID-19 Competitive Revision Award' linked with grant no. DP5OD028123 (principal investigator: J.L.S.) to J.L.S. J.L.S. also receives grant support from the National Institutes of Health (DP5OD28123), the Klingenstein Third Generation Foundation, the American Psychological Foundation, the Society for Clinical Child and Adolescent Psychology, and Limbix, Inc. J.L.S. and K.R.F. receive grant support from the Upswing Fund for Adolescent Mental Health. M.L.D. receives grant support from a Stony Brook University Graduate Research Fellowship and the Psi Chi Honor Society. J.L.S., M.L.D. and M.C.M. have co-authored and receive royalties from sales of a therapeutic workbook for adolescents, published by New Harbinger. J.L.S. is under 
contract with Oxford University Press to co-edit a book on low-intensity mental health interventions for youth. The funders had no role in study design, data collection and analysis, decision to publish or preparation of the manuscript.

\section{Author contributions}

J.L.S. conceptualized the project, acquired the funding and wrote the original manuscript draft. J.L.S., M.C.M. and K.R.F. contributed to the methodology and study design. All authors contributed to the review and editing of the final manuscript. K.R.F. and E.A.H. provided resources critical to study execution. A.S., C.A.R., M.C.M., K.R.F., E.A.H. and J.L.S. contributed to data curation. J.L.S., K.R.F., C.A.R., A.S. and E.A.H. contributed to project administration. M.C.M. and M.L.D. wrote the analytic code. M.C.M. created the tables and visualizations and conducted the formal analysis. M.L.D. validated the study results.

\section{Competing interests}

The authors delare no competing interests.

\section{Additional information}

Supplementary information The online version contains supplementary material available at https://doi.org/10.1038/s41562-021-01235-0.

Correspondence and requests for materials should be addressed to Jessica L. Schleider.

Peer review information Nature Human Behaviour thanks Robert DeRubeis and

Simon Rice for their contribution to the peer review of this work. Peer reviewer reports are available.

Reprints and permissions information is available at www.nature.com/reprints.

Publisher's note Springer Nature remains neutral with regard to jurisdictional claims in published maps and institutional affiliations.

ClinicalTrials.gov Identifier: NCT04634903; Open Science Framework pre-registration: osf.io/kumdv

(C) The Author(s), under exclusive licence to Springer Nature Limited 2021 


\section{Reporting Summary}

Nature Research wishes to improve the reproducibility of the work that we publish. This form provides structure for consistency and transparency in reporting. For further information on Nature Research policies, see our Editorial Policies and the Editorial Policy Checklist.

\section{Statistics}

For all statistical analyses, confirm that the following items are present in the figure legend, table legend, main text, or Methods section.

n/a Confirmed

$\square$ The exact sample size $(n)$ for each experimental group/condition, given as a discrete number and unit of measurement

$\square$ \A statement on whether measurements were taken from distinct samples or whether the same sample was measured repeatedly

The statistical test(s) used AND whether they are one- or two-sided

$\square$ Only common tests should be described solely by name; describe more complex techniques in the Methods section.

$\square$ \ A description of all covariates tested

$\square$ \A description of any assumptions or corrections, such as tests of normality and adjustment for multiple comparisons

$\square$ A full description of the statistical parameters including central tendency (e.g. means) or other basic estimates (e.g. regression coefficient)

$\triangle$ AND variation (e.g. standard deviation) or associated estimates of uncertainty (e.g. confidence intervals)

$\varnothing$ For null hypothesis testing, the test statistic (e.g. $F, t, r$ ) with confidence intervals, effect sizes, degrees of freedom and $P$ value noted

Give P values as exact values whenever suitable.

Х $\square$ For Bayesian analysis, information on the choice of priors and Markov chain Monte Carlo settings

Х $\square$ For hierarchical and complex designs, identification of the appropriate level for tests and full reporting of outcomes

$\square \bigotimes$ Estimates of effect sizes (e.g. Cohen's $d$, Pearson's $r$ ), indicating how they were calculated

Our web collection on statistics for biologists contains articles on many of the points above.

\section{Software and code}

Policy information about availability of computer code

Data collection Qualtrics Version 2020

Data analysis R Version 4.0.3 (Reproduced in R Version 4.0.0)

For manuscripts utilizing custom algorithms or software that are central to the research but not yet described in published literature, software must be made available to editors and reviewers. We strongly encourage code deposition in a community repository (e.g. GitHub). See the Nature Research guidelines for submitting code \& software for further information.

\section{Data}

Policy information about availability of data

All manuscripts must include a data availability statement. This statement should provide the following information, where applicable:

- Accession codes, unique identifiers, or web links for publicly available datasets

- A list of figures that have associated raw data

- A description of any restrictions on data availability

The deidentified data is available on the Open Science Framework at https://osf.io/r68xj/ 


\section{Field-specific reporting}

Please select the one below that is the best fit for your research. If you are not sure, read the appropriate sections before making your selection.
Life sciences
Вehavioural \& social sciences
Ecological, evolutionary \& environmental sciences

For a reference copy of the document with all sections, see nature.com/documents/nr-reporting-summary-flat.pdf

\section{Behavioural \& social sciences study design}

All studies must disclose on these points even when the disclosure is negative.

Study description

Research sample

Sampling strategy

Data collection

Timing

Data exclusions

Non-participation

Randomization
Quantitative randomized control trial

The sample is $13-16$ year old adolescents (Sex at Birth $=80 \%$ Female, Identify as BIPOC $=33.45 \%$ ) who screened positive for major depressive disorder based on scoring $>=2$ on the PHQ-2 screener. The sample was nationwide in the USA but not representative, as we prioritized enrolling participants who screened positive for depression.

We conducted an a priori power analysis using the pwr package in $\mathrm{R}$ that found with 800 participants per randomized group we would have $98 \%$ power to detect a difference of $d=0.20$. Sampling was nationwide (USA), online, and only included adolescents who screened positive for major depressive disorder. Therefore, the sample was nationwide (USA) but not representative.

The data collection procedure occurred entirely online (phone, tablet, computer, or any internet-connected device), and the participants/researchers were masked to condition assignment as the randomization occurred automatically within the Qualtrics survey platform. Participants were encourage to complete the study in a place with sufficient physical privacy for their comfort.

November 19 - December 6, 2020 = Baseline data collection

February 11 - March 15, 2021 = Follow-up data collection

All randomized participants were analyzed using multiple imputation strategies

1,285 youth declined to participate after meeting inclusion criteria, and a total of 1,399 exited the survey prior to randomization

The randomization occurred automatically within the Qualtrics survey platform.

\section{Reporting for specific materials, systems and methods}

We require information from authors about some types of materials, experimental systems and methods used in many studies. Here, indicate whether each material, system or method listed is relevant to your study. If you are not sure if a list item applies to your research, read the appropriate section before selecting a response.

Materials \& experimental systems

\begin{tabular}{l|l}
\hline$n / a$ & Involved in the study \\
$\square$ & $\square$ Antibodies \\
$\square$ & $\square$ Eukaryotic cell lines \\
$\square$ & $\square$ Palaeontology and archaeology \\
$\square$ & $\square$ Animals and other organisms \\
$\square$ & $\bigotimes$ Human research participants \\
$\square$ & $\bigotimes$ Clinical data \\
$\square$ & $\square$ Dual use research of concern
\end{tabular}

\begin{tabular}{l|l} 
Methods \\
\hline n/a & Involved in the study \\
$\bigotimes$ & $\square$ ChIP-seq \\
$\bigotimes$ & $\square$ Flow cytometry \\
$\bigotimes$ & $\square$ MRI-based neuroimaging
\end{tabular}

\section{Human research participants}

Policy information about studies involving human research participants

Population characteristics

See above

Recruitment

Participants learned about the study through Instagram advertisements following established ethics guidelines for passive, social media based recruitment. Posts included invitations to determine eligibility for a confidential, online psychology study, for which participants could earn up to \$20 USD in gift cards. Instagram posts linked to a Qualtrics survey; the first page included study details and an invitation to complete an eligibility screener. 
Policy information about clinical studies

All manuscripts should comply with the ICMJE guidelines for publication of clinical research and a completed CONSORT checklist must be included with all submissions.

Clinical trial registration Clinicaltrials.gov identifier: NCT04634903

Study protocol

Clinicaltrials.gov identifier: NCT04634903

Data collection

The data collection procedure occurred entirely online (phone, tablet, computer, or any internet-connected device), and the participants/researchers were masked to condition assignment as the randomization occurred automatically within the Qualtrics survey platform. Participants were encourage to complete the study in a place with sufficient physical privacy for their comfort.

November 19 - December 6, 2020 = Baseline data collection

February 11 - March 15, 2021 = Follow-up data collection

Outcomes

See Clinicaltrials.gov identifier: NCT04634903 